\title{
Determination of the relationship between Vietnam national coordinate reference system (VN-2000) and ITRS, WGS84 and PZ-90
}

\author{
Hoa Pham Thi ${ }^{1 *}$, Dung Nghiem Quoc ${ }^{2}$, Thu Trinh Thi Hoai ${ }^{1}$, Huynh Pham The ${ }^{3}$ and Nhung Le Thi ${ }^{1}$

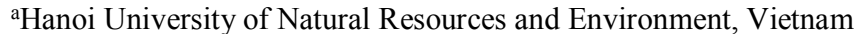 \\ ${ }^{\mathrm{b}}$ Department of Surveying, Mapping and Geoinformation of Vietnam, Vietnam \\ ${ }^{\mathrm{c}}$ Hanoi University of Mining and Geology, Vietnam
}

\begin{abstract}
In July 2000, Hanoi-72 reference system was replaced by the Vietnam reference system, namely as VN-2000 as an official geodetic background system in Vietnam. Ministry of Natural Resources and Environment of Vietnam has reported the transformation parameters between VN2000 and WGS84. Nevertheless, there is a need to estimate a new transformation parameter set between VN-2000 and WGS84 because WGS84 has been updated. In addition, there is now a lack of an accurate published set of parameters for transformation from VN-2000 to not only the International Terrestrial Reference System ITRS but also PZ-90. In this study, coordinate transformation parameters between ITRS and VN-2000 are estimated through the use of a least square approach and the common points with known coordinates in both systems. These set of parameters was then deployed to determine the link between VN-2000 and WGS84 as well as PZ90. The results denoted that the derived transformation parameters, on the basis of the results at the checkpoints, could generated station positions with the accuracy at several $\mathrm{cm}$ level for transformation from VN-2000 to the new realizations of ITRS, WGS84 and PZ90 and reversely. These achievements reveals that the set of parameters is great significance for many applications related to positioning in Vietnam.
\end{abstract}

Keywords: VN-2000, ITRS, ITRF, WGS84, PZ-90, coordinate transformation parameters

\section{Introduction}

In the era of globalization and modernization, Vietnam, a developing country, would adopts many scientific and technological achievements. In which the use of geospatial data generated by countries with the most advanced technology is extremely useful. However, these data sources are not in VN-2000 but in different coordinate systems. Thus, there is a need to convert spatial data in disparate sources to VN-2000 and and reversely.

With the Decree of the Vietnam Government No.83 dated December 12, Vietnam national coordinate reference system ( VN-2000) became an became the official national reference system [1]. VN-2000 is static, or plate- fixed reference system, so its coordinates do not constantly change with time. The concept of a static datum, like VN-2000, has served Vietnam and many other countries well because it is easier to implement and maintain than dynamic coordinate systems. However, the dynamic one enables the change of positions on the earth to be tracked and modeled over time.

Ministry of Natural Resources and Environment of the Socialist Republic of Vietnam (Monre) has reported the transformation parameters between the VN-2000 and World Geodetic System 1984 (WGS84) [2]. Nevertheless, there is a need to estimate a new transformation parameter set between two systems because WGS84 has been updated.
Nowadays, the International Terrestrial Reference systems (ITRS) is more widely used than WGS84 [3]. However, there is now no means of transforming ITRF coordinate values to the local system. The lack of transformation parameters causes inconvenience to the Vietnamese geodetic community, especially the National GNSS CORS network is being established in Vietnam and will have been connected to the IGS network. Therefore, its stations will have accurate ITRF-based coordinates. Definitely, to meet regulatory requirements, data must be provided in the official datum. Therefore, it is necessary to have a readily available parameter sets for ITRF to VN2000 transformations.

Along with the US GPS system using WGS84, the Russia Glonass system using the PZ- 90 reference system also could operate independently and promising more potential applications in Vietnam. However, the PZ-90, like ITRS, has no connection with VN-2000.

The shortage of official links between VN-2000 with three above-mentioned international reference systems will make VN-2000-based data not be able to integrate with international ones. Therefore, this study, as an interim method, aims to define the relationship between the ITRS and VN-2000 based the use of a least square approach and common points with known coordinates in both systems. Then, transformation parameters between the VN-2000 and the ITRS are used to determine the links between VN-2000 and not only WGS84 but also PZ-90. 


\section{Overview of $\mathrm{VN}-2000$, WGS84, PZ-90 and ITRS}

\subsection{Overview of VN-2000}

Since 1990, with the rapid development of satellite- based positioning technology in terms of accuracy and accessibility, Vietnam built new networks across the country and these networks was connected with older ones. Based on the data collection of networks across the country over time, from 1998 to 1999 , the Viet nam General Department of Land Administration has deployed the project "Construction of the national coordinate system -VN-2000" [4]. The project was implemented 1999 and VN-2000 have been officially the Vietnam national reference system from July 2000 to date.

The classical VN-2000 datum is not geocentric. It is based on the ellipsoid which derived from fitting WGS84 reference ellipsoid to local geoid based on a least squares method [4]. VN-2000 is static, so there is no requirement for epoch of its coordinates. Further detailed information on the observation and adjustment of VN-2000 was described by [4].

\subsection{Overview of ITRS}

The ITRS is a system defined by the International Association of Geodesy and the International Astronomical Union and endorsed by the International Union of Geodesy and Geophysics [5]. The ITRS is geocentric and dynamic. The practical realization of ITRS is the International Terrestrial Reference Frame (ITRF). The ITRF was first published in 1988 and is the most precise earth-centered, earth-fixed coordinate fame currently available. It is maintained by the International Earth Rotation and Reference Systems Service and realized by an extensive global network of accurate coordinates derived from geodetic observations using GPS, Very Long Baseline Interferometry (VLBI), Satellite Laser Ranging (SLR), Lunar Laser Ranging (LLR) and Doppler Orbitography and Radiopositioning Integrated by Satellite (DORIS) [6]. To date, there are many ITRF realizations, for example, ITRF1992 [7], ITRF1996 [8], ITRF1997 [9], ITRF2000 [10], ITRF2005 [6], ITRF2008 [11] and most recently ITRF2014 [12]. Transformation parameters among ITRF products are availabe on the offical website [13].

The ITRF is a common standard which warrants interoperability between geospatial information systems. There are some main reasons for this. Firstly, most regional and global reference frames are today aligned or related to ITRF [5]. Secondly, many countries have redefined their geodetic systems to be compatible with ITRF [5]. Thirdly, all global satellite positioning systems can easily be used to coordinates in a recent ITRF [5]. Finally, all GNSS networks around the world can easily be expressed in the ITRF using IGS products [5]. As a consequence of these reasons, ITRF is becoming the most widely used international coordinate system. This calls for the production of a mathematical model of transformation from ITRF to VN-2000 and reversely. The availability of transformations between the two systems enables Vietnam users to enhance interoperability of geo-referenced datasets and services towards homogeneity of spatial data. More information on ITRS is available in [14].

\subsection{Overview of WGS84 and PZ-90}

The WGS84 and PZ-90 represent the system of geodetic parameters including fundamental geodetic constants, reference ellipsoid parameters, the Earth's gravity field parameters, geocentric coordinate system and transformation parameters to other reference systems.

WGS84 is a conventional Earth reference system established by the US Department of Defense (DoD), and used as the nominal system for GPS navigation [14]. The original WGS84 reference frame established in 1987, commonly known as WGS84 (Doppler), based on U.S. Navy Navigation Satellite System or TRANSIT (Doppler). It generated station coordinates with accuracies of about one meter. The others realizations of WGS84 based on GPS data, such as WGS84 (G730), WGS84 (G873), WGS84 (G1150), WGS84 (G1674), WGS84 (G1762), where the ' $G$ ' indicates these coordinates were obtained through GPS techniques and the number following the ' $G$ ' indicates the GPS week number when these coordinates were implemented in the precise GPS ephemeris estimation process [14]. The WGS84 (G730) reference frame are coincident with ITRF 92 at about 10-centimeter level [15]. However, the lastest G1762 realization adopted ITRF2008 coordinates for more than half of the reference stations and velocities of nearby sites for the others. Thus, ITRF2014, ITRF2008 and WGS84 (G1762) are in agreement at the centimeter level, provided conventional 0 -transformation parameters. More information on WGS84 is available in [14].

The PZ-90 is a global system developed by Russia, similar to WGS84, and is a basis for geodetic support of the Global Navigation Satellite System (GLONASS). Up to now, the PZ 90 reference system has undergone two modernizations with corresponding versions of PZ 90.00, PZ-90.02 and PZ-90.11 [16]. In PZ-90.11 axes orientation, scale and origin are coincided to the similar ITRF parameters at the $\mathrm{cm}$ level. Detailed information on PZ-90 is available in the document [16].

WGS84, PZ-90 and ITRS were determined based on the same theoretical principles. However, there are small differences between the three's practical realizations that can be explained by discrepancy not only in the number, categories and distribution of input data but also in methodological differences [16]. Coordinate transformation between products of these references system are available in [14][16].

\section{Coordinate transformation method}

The most common used methodology to convert coordinate from one system to the other deploys a least square principle and known fundamental points, whose coordinates are known on both systems. However, in Vietnam, high accuracy VN-2000 and ITRF coordinates can be determined, but not WGS84 and PZ-90 coordinates [5]. Thus, this paper employs a least square method to deliver VN-2000 coordinates from an ITRF based computation system and then used relationship between ITRS and the others systems for estimation of 
parameters for coordinate transformation from WGS84 and PZ-90 to VN-2000.

\subsection{ITRS to $\mathrm{VN}-2000$ coordinate transformation method}

Coordinates given with respect to $\mathrm{VN}-2000$ are time independent. In contrast, coordinates given with respect to ITRF are time dependent. Conversely, the International Terrestrial Reference Frame (ITRF) is dynamic, its coordinates change as linear functions of time due to tectonic motion [17]. Therefore, the transformation approach between the two systems is to use a 14 parameter transformation, which augment the original 7 parameters (three translations, three rotations, and one scale factor) with their time derivatives. This approach is currently applied in Australia to transform ITRF coordinates at the measurement epoch to Australian coordinate system (GDA94) which is a static datum fixed with ITRF92 at epoch 1994.0 [18]. It is also used in other countries and region such as Europe [19], United States, Canada, Mexico, Central America and the Caribbean Islands [20].

The general formulation to transform coordinates between two systems, say ITRF to VN-2000, is given according to the following equations [21]

$$
X_{(2)}=T+(1+D) R^{T} X_{(1)},
$$

where $\mathrm{X}_{(2)}$ is the vector of $\mathrm{VN}-2000$-based coordinates, $\mathrm{X}_{(1)}$ is the vector of ITRF-based coordinates; $\mathrm{T}$ is the vector translation components, $\mathrm{D}$ is the differential scale change, $\mathrm{R}$ is the matrix of differential rotation angles (expressed in radians) as follows [21]:

$$
{ }^{[\mathrm{A} 1]} R^{T}=\left(\begin{array}{rcc}
1 & -R_{3} & R_{2} \\
R_{3} & 1 & -R_{1} \\
-R_{2} & R_{1} & 1
\end{array}\right)
$$

Because D is small quantity, we can neglect secondorder terms. Equation (1) can be express as follows [22]:

$\left(\begin{array}{l}X \\ Y \\ Z\end{array}\right)_{(1)}=\left(\begin{array}{l}X \\ Y \\ Z\end{array}\right)_{(1)}+\left(\begin{array}{l}T_{1} \\ T_{2} \\ T_{3}\end{array}\right)_{(1)}+D\left(\begin{array}{l}X \\ Y \\ Z\end{array}\right)_{(1)}+\left(\begin{array}{ccc}0 & -R_{3} & R_{2} \\ R_{3} & 0 & -R_{3} \\ R_{2} & R_{1} & 0\end{array}\right)\left(\begin{array}{l}X \\ Y \\ Z\end{array}\right)_{(1)}$

The seven transformation parameters, T1, T2, T3, R1, R2, R3 and D, change with time, thus, (1) can be expressed in other form as [21]:

$$
\begin{aligned}
& {\left[\begin{array}{c}
X(t) \\
Y(t) \\
Z(t)
\end{array}\right]_{(2)}=\left[\begin{array}{c}
X(t) \\
Y(t) \\
Z(t)
\end{array}\right]_{(1)}+\left[\begin{array}{l}
T_{1}\left(t_{0}\right)+Z_{1}^{\&}\left(t-t_{0}\right) \\
T_{2}\left(t_{0}\right)+Z_{2}^{\&}\left(t-t_{0}\right) \\
T_{3}\left(t_{0}\right)+Z_{3}^{\&}\left(t-t_{0}\right)
\end{array}\right]_{(1)}} \\
& +\left(D\left(t_{0}\right)+B\left(t-t_{0}\right)\right)\left[\begin{array}{c}
X(t) \\
Y(t) \\
Z(t)
\end{array}\right]_{(1)}+\tilde{R}\left[\begin{array}{c}
X(t) \\
Y(t) \\
Z(t)
\end{array}\right]_{(1)},
\end{aligned}
$$

where $t$ is computed epoch (yr), $t_{0}$ is the reference epoch (yr) of the transformation parameters and:

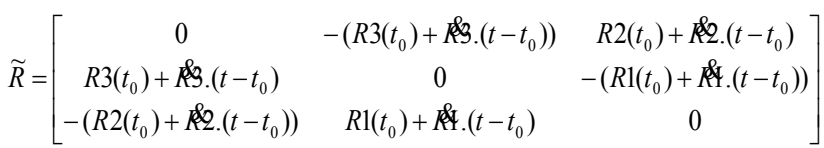

Differentiating Equation (1) with respect to time and eliminating the negligible terms it can be written [21]

$$
X_{2}^{\&}=X_{1}^{\&}+\not \&+B X_{1}+D X_{1}^{\&}+R X_{1}+R X_{1}^{\&},
$$

where $X_{2}^{\&}, X_{1}^{\&}, T_{,}^{\&}, \mathbb{R}$ is the time derivatives of $X_{2}, X_{1}, T, D, R$, respectively.

Since D and R is very small, hence Equation (5) can be reduced to [21]:

$$
\not X_{2}^{\&}=\not X_{1}^{\&}+\not \&+\& X_{1}+\not X_{1}
$$

To estimate the fourteen- parameter similarity transformation between the two system, the coordinate differences between the ITRF and VN-2000 were treated as observations in the least squares solution of equations. Therefore, the identical points are used in a least squares process to define the relationship between the two systems. The above equations can be written as [21]:

$$
\begin{aligned}
& X_{2}=X_{1}+T+D X_{1}+R X_{1} \Leftrightarrow X_{2}=X_{1}+A \theta, \\
& X_{2}^{\&}=X_{1}^{\&}+X^{\&}+B X_{1}+K_{1}^{\&} \Leftrightarrow X_{2}^{\&}=X_{1}^{\&}+A \&, \\
& \theta=\left[T_{1}, T_{2}, T_{3}, D, R_{1}, R_{2}, R_{3}\right] \\
& \theta=\left[T_{1}^{\&}, \mathbb{T}_{2}^{\&}, \mathbb{R}_{3}^{\&}, \mathcal{B}, \mathbb{K}_{1}^{\&}, \mathbb{R}_{2}^{\&}, \mathbb{K}_{3}^{\&}\right]
\end{aligned}
$$

with:

The least squares solutions are:

$$
\begin{aligned}
& \theta=\left(A^{T} P_{x} A\right)^{-1} A^{T} P_{x}\left(X_{2}-X_{1}\right), \\
& \& \&=\left(A^{T} P_{v} A\right)^{-1} A^{T} P_{v}\left(X_{2}^{\&}-X_{1}^{\&}\right),
\end{aligned}
$$

where $P_{X}$ and $P_{v}$ are the weight matrix for station positions and velocities, respectively.

The statistical calculation a posterior variance of unit weight is determined as follows [21]:

$$
s_{0 X}^{2}=\left(\left(A^{T} P_{x}-\left(X_{2}-X_{1}\right)\right)^{-1} \cdot\left(A^{T} P_{x}-\left(X_{2}-X_{1}\right)\right)^{-1}\right) / n
$$

where $\mathrm{n}$ is degrees of freedom.

The above mentioned principle derives parameters for a transformation between ITRF products and VN-2000 through the use of a least square approach and common points with known coordinates in both systems. The achievable accuracy of the transformation parameters is depended on the number, distribution and accuracy of the common points. It is necessary to obtain far more than the minimum number of common points because the redundant points will give an idea of the consistency of the derived transformation parameters. The accuracy of these parameters should be tested by comparing actual and transformed positions of check points.

The process of estimation coordinate transformation parameters between ITRF and VN-2000 consists of three steps following: 
-Step 1: Identify stations at which coordinates are available in both the reference frames and velocities are available in ITRF

-Step2:Calculate appropriate coordinate transformation parameters

-Step 3:Assess the accuracy and reliability of the achieving transformation parameters based on using checkpoints as an independent check by comparing actual and transformed positions.

\subsection{WGS84 and PZ-90 to VN-2000 coordinate transformation methods}

As mentioned before, due to difficulty in determining WGS84 and PZ-90 coordinates with high accuracy in Vietnam, the paper proposed coordinate convertion from WGS84 and PZ-90 to VN-2000 based on relationship between ITRF and the three as the following formula:

$\mathrm{VN}-2000 \rightarrow$ WGS $84=(\mathrm{VN}-2000 \rightarrow \mathrm{ITRF})+(\mathrm{ITRF} \rightarrow \mathrm{WGS} 84)$

$\mathrm{VN}-2000 \rightarrow \mathrm{PZ}-90=(\mathrm{VN}-2000 \rightarrow \mathrm{ITRF})+(\mathrm{ITRF} \rightarrow \mathrm{PZ}-90)$

\section{Input solution datasets}

To define transformation from WGS84, PZ-90 and ITRF to VN-2000, data from two networks was collected. The first network belongs to the annual Asia Pacific Regional Geodetic Project (APRGP) GPS campaign, which are an important activity of the regional geodesy working group of the Permanent Committee on GIS Infrastructure for Asia and the Pacific Region (PCGIAP) [23,24]. This network consists of 7 -day observation sessions and have been undertaken from 1997 to 2016. The GPS data were processed using the Bernese GPS Software in a regional network together with selected IGS (International GNSS Service) sites [23]. The GPS solution was constrained to the ITRF2008 reference frame through adopting IGS08 coordinates on selected IGS reference sites and using the final IGS earth orientation parameters and ephemerides products[23].The second network is the Vietnamese marine one, which also consists of 7-day observation sessions and have been accomplished in 2016. Its GPS observation data were processed to deliver ITRF2005based coordinates at epoch 2016.764 and VN-2000 coordinate values.

This research used data from a total of 21 stations within the two above mentioned networks, and their coordinates available in both ITRS and VN-2000 coordinate systems. Of these, ten well distributed common points in Vietnam, belonging to (APRGP) GPS campaign are used to compute transformation parameters between ITRS and VN-2000. Their ITRF2008-based coordinates and velocities were collected over a period of 4 years (2011-2015) [24, 25, 26,27]. Their VN-2000 coordinate values obtained by connecting them with other GPS local networks. The left eleven points belonging to the Vietnamese marine network was used as independent check points for assessing of achieved parameter accuracy.

The set of transfromation parameters between ITRS and VN-2000 was then deployed to determine the link between VN-2000 and WGS84 as well as PZ-90. The both networks were also employed to test parameters for transformation from WGS84 and PZ-90 to VN-2000.
The station positions are shown in Fig.1.

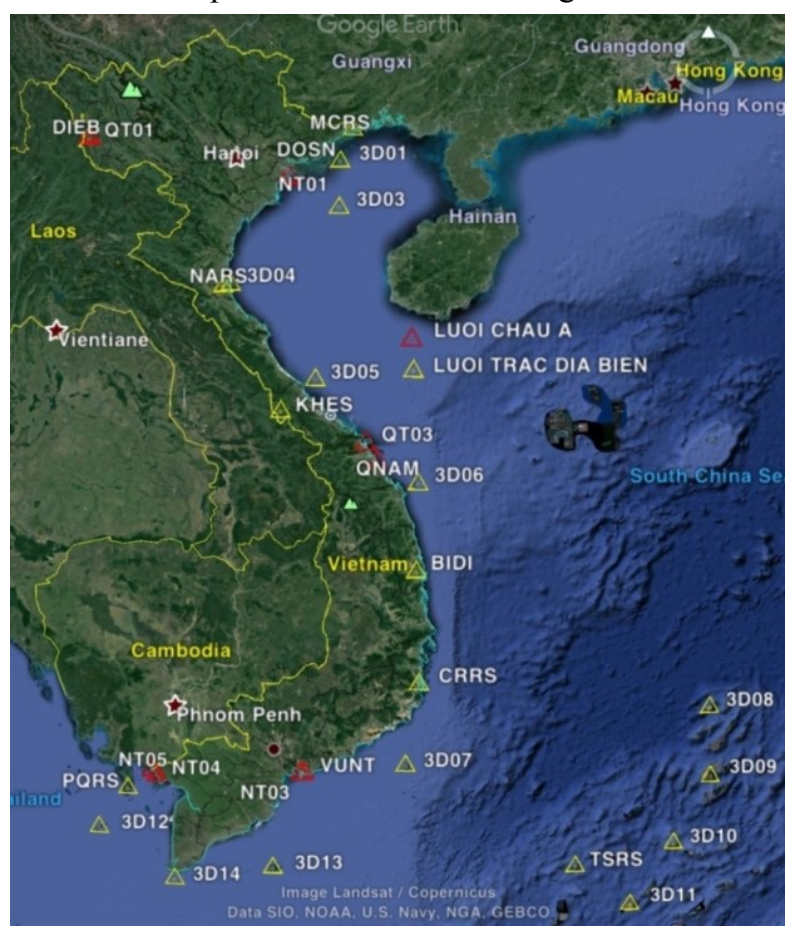

Fig.1 The position of 21 stations in two networks.

\section{Results and discussion}

\subsection{Transfromation from ITRF to VN-2000}

Originally, coordinates and velocities at 10 points in $A P R G P$, known on the ITRF2008 and VN-2000, were used to determine the transformation parameters at epoch 2015.0. The defined parameters for transforming ITRF2008 to VN-2000 are shown in Table 1. The statistical a posterior variance of unit weight are $4.67 \mathrm{~cm}$ and $0.52 \mathrm{~cm} / \mathrm{yr}$ for original 7 parameters and their rates, respectively.

The transformation parameters between the other ITRF realizations denoted by ITRF $\mathrm{XY}_{\mathrm{XY}}$ and $\mathrm{VN}-2000$ is determined according to the following formula:

$I T R F_{x y} \rightarrow \mathrm{VN} 2000=\left(I T R F_{x y} \rightarrow I T R F 2008\right)+(I T R F 2008 \rightarrow V N 2000)$

The additional required information for propagating from ITRF $F_{X Y}$ to ITRF2008 to complete the transformation from ITRF $\mathrm{IXY}_{\mathrm{X}}$ to VN-2000 are published in the IERS Technical Notes and Annual Reports and available on the official ITRF webpage [13]. The final values of the 14 transformation parameters to transform coordinates given on the ITR $\mathrm{XY}_{\mathrm{XY}}$ to the VN-2000 are presented in Table 1. Table 1 shows that two origins of systems have positive displacements on all the axes. The negative scale factor values indicate that the geometric shapes of the ITRF and VN-2000 are reduced when the scale factor is applied in conjunction with the other parameters for transforming coordinates from ITRF to VN-2000.

ITRF use the Geodetic Reference System 1980 (GRS80) as the reference ellipse to represent geographical coordinates whereas VN-2000 adopts the WGS84 reference ellipse that is slightly different to GRS80. However, the translations in table 3 are very significant and must be considered because the WGS84 reference 
ellipse in VN-2000 was best fit with local geoid. In general, these parameter values are not much different compared with the set of transformation parameters between WGS84 and VN-2000, and it is understood because WGS84 is regularly aligned to ITRF $[5,28]$.

Table 1. Transformation parameters from ITRFXY to VN-2000 at epoch 2015.0. Units are in $\mathrm{m}$ and $\mathrm{m} . \mathrm{yr}^{-1}$ for the translations and their rates, respectively, parts-per-billion (ppb) and ppb.yr ${ }^{-1}$ for scale and its rate, respectively, and milli-arcseconds (mas) and mas. $\mathrm{yr}^{-1}$ for the rotations and their rates, respectively.

\begin{tabular}{|c|c|c|c|c|c|c|}
\hline $\begin{array}{l}\mathrm{T}_{1} \\
T_{1}^{\&}\end{array}$ & $\begin{array}{l}\mathrm{T}_{2} \\
T_{2} \&\end{array}$ & $\begin{array}{l}\mathrm{T}_{3} \\
T_{3}^{\&}\end{array}$ & $\begin{array}{l}\mathrm{D} \\
\& \\
\&\end{array}$ & $\begin{array}{l}\mathrm{R}_{1} \\
R_{1}^{\&}\end{array}$ & $\begin{array}{l}\mathrm{R}_{2} \\
\mathbb{R}_{2}^{\&}\end{array}$ & $\begin{array}{l}\mathrm{R}_{3} \\
R_{3}^{\&}\end{array}$ \\
\hline \multicolumn{7}{|l|}{ ITRF2014 } \\
\hline 193.9227 & 37.5110 & 110.6343 & -7.51 & -7.11 & +20.08 & +37.35 \\
\hline-0.0790 & -0.0360 & 0.0188 & 0.16 & -0.85 & +1.33 & -3.52 \\
\hline \multicolumn{7}{|l|}{ ITRF2008 } \\
\hline 193.9211 & 37.5091 & 110.6319 & -7.54 & -7.11 & +20.08 & +37.35 \\
\hline-0.0790 & -0.0360 & 0.0189 & 0.16 & -0.85 & +1.33 & -3.52 \\
\hline \multicolumn{7}{|l|}{ ITRF2005 } \\
\hline 193.9186 & 37.5100 & 110.6366 & -8.48 & -7.11 & +20.08 & +37.35 \\
\hline-0.0793 & -0.0360 & 0.0189 & 0.16 & -0.85 & +1.33 & -3.52 \\
\hline \multicolumn{7}{|l|}{ ITRF2000 } \\
\hline 193.9215 & 193.9213 & 110.6694 & -10.08 & -7.11 & +20.08 & +37.35 \\
\hline-0.0791 & -0.0361 & 0.0207 & 0.08 & -0.85 & +1.33 & -3.52 \\
\hline \multicolumn{7}{|l|}{ ITRF97 } \\
\hline 193.9148 & 193.9260 & 110.7131 & -11.81 & $-7,11$ & $+20,08$ & $+36,99$ \\
\hline-0.0791 & -0.0355 & 0.0221 & 0.07 & $-0,85$ & $+1,33$ & $-3,54$ \\
\hline \multicolumn{7}{|l|}{ ITRF96 } \\
\hline 193.9148 & 193.9260 & 110.7131 & -11.81 & $-7,11$ & $+20,08$ & $+36,99$ \\
\hline-0.0791 & -0.0355 & 0.0221 & 0.07 & $-0,85$ & $+1,33$ & $-3,54$ \\
\hline \multicolumn{7}{|l|}{ ITRF94 } \\
\hline 193.9148 & 193.9260 & 110.7131 & -11.81 & $-7,11$ & $+20,08$ & $+36,99$ \\
\hline-0.0791 & -0.0355 & 0.0221 & 0.07 & $-0,85$ & $+1,33$ & $-3,54$ \\
\hline \multicolumn{7}{|l|}{ ITRF93 } \\
\hline 193,9871 & 193,9202 & 110,7065 & $-12,30$ & $-3,75$ & 24,41 & 36,60 \\
\hline$-0,0762$ & $-0,0359$ & 0,0213 & 0,07 & $-0,74$ & 1,52 & $-3,59$ \\
\hline \multicolumn{7}{|l|}{ ITRF92 } \\
\hline 193,9068 & 193,9240 & 110,7211 & $-11,10$ & $-7,11$ & 20,08 & 36,99 \\
\hline$-0,0791$ & $-0,0355$ & 0,0221 & 0,07 & $-0,85$ & 1,33 & $-3,54$ \\
\hline \multicolumn{7}{|l|}{ ITRF91 } \\
\hline 193,8948 & 193,9100 & 110,7271 & $-12,50$ & $-7,11$ & 20,08 & 36,99 \\
\hline$-0,0791$ & $-0,0355$ & 0,0221 & 0,07 & $-0,85$ & 1,33 & $-3,54$ \\
\hline \multicolumn{7}{|l|}{ ITRF90 } \\
\hline 193,4813 & 193,3275 & 112,2131 & 121,10 & $-7,11$ & 20,08 & 36,39 \\
\hline$-0,0791$ & $-0,0355$ & 0,0221 & 0,07 & $-0,85$ & 1,33 & $-3,54$ \\
\hline \multicolumn{7}{|l|}{ ITRF89 } \\
\hline 193,5513 & 193,8435 & 112,6111 & 171,00 & $-8,61$ & 20,08 & 36,39 \\
\hline$-0,0791$ & $-0,0355$ & 0,0221 & 0,07 & $-0,85$ & 1,33 & $-3,54$ \\
\hline \multicolumn{7}{|l|}{ ITRF88 } \\
\hline 193,8983 & 193,9185 & 110,7571 & $-17,95$ & $-7,21$ & 20,08 & 37,29 \\
\hline$-0,0791$ & $-0,0355$ & 0,0221 & 0,07 & $-0,85$ & 1,33 & $-3,54$ \\
\hline
\end{tabular}

The transformation is done in a single process, enabling ITRF $F_{X Y}$ data at any epoch (denoted as t) to be transformed to $\mathrm{VN}-2000$ based on a 14-parameter transformation as the following two steps:

- Step 1: Calculate transformation parameters between ITRF $_{X Y}$ and VN-2000 at Epoch t utilizing data in table 2

- Step 2: Calculate coordinates at Epoch $t$ based on the achieved parameters.

Table 3 The differences between the computed and existing VN-2000 coordinates of checkpoints

\begin{tabular}{cccc} 
Points & $\begin{array}{c}\text { Diff. X } \\
(\mathrm{m})\end{array}$ & $\begin{array}{c}\text { Diff.Y } \\
(\mathrm{m})\end{array}$ & $\begin{array}{c}\text { Diff. Z } \\
(\mathrm{m})\end{array}$ \\
\hline 3D01 & +0.049 & +0.011 & +0.004 \\
3D02 & +0.040 & +0.015 & +0.008 \\
3D03 & +0.060 & -0.076 & -0.031 \\
3D04 & +0.058 & -0.012 & -0.003 \\
3D05 & +0.047 & -0.037 & -0.029 \\
3D06 & +0.028 & -0.026 & -0.035 \\
3D07 & +0.103 & -0.142 & -0.068 \\
3D13 & +0.088 & -0.049 & -0.045 \\
3D14 & +0.045 & +0.093 & -0.009 \\
BIDI & +0.045 & -0.052 & -0.058 \\
KHES & +0.040 & -0.015 & -0.025 \\
Max Diff. & +0.103 & +0.093 & +0.008 \\
Min Diff. & +0.028 & -0.142 & -0.068 \\
Mean Diff. & +0.055 & -0.026 & -0.026 \\
Standard Diff. & \pm 0.059 & \pm 0.062 & \pm 0.035
\end{tabular}

To carry out the test of the computed transformation parameters, available ITRF2005 and VN-2000 coordinates of eleven points within marine networks that did not participate in the transformation solution were utilized. The 11 selected are 3D01, 3D02, 3D03, 3D04, 3D05, 3D07, 3D13, 3D14, BIDI and KHES. The test was directly performed using data in table 2 to transfer ITRF2005based to VN-2000 coordinates. The differences between the VN-2000 values, after conversion, and the existing VN-2000 X, Y and Z values of independent test points are tabulated in Table 3.

These coordinate differences should ideally be zero. However, such results could never be achieved because of the effect of input data uncertainty. Data in table 3 indicates that transformation $\mathrm{Z}$ coordinates gave a better accuracy compared to the $\mathrm{X}$ and $\mathrm{Y}$ components. The standard deviation is approximate $6 \mathrm{~cm}$ for $\mathrm{X}$ and $\mathrm{Y}$ whereas $3.5 \mathrm{~cm}$ to $Z$. The coordinate difference is within 5 $\mathrm{cm}$ for $100 \%$ points in term of $\mathrm{Z}$ whereas $82.1 \%$ points in $\mathrm{X}$ and $\mathrm{Y}$.



Fig 2. Coordinate Differences 
The computed coordinate differences of checkpoints in Tables 3, represented in Fig. 2 clearly reflects residuals for the eleven test points.

Fig. 2 shows that the coordinate differences fluctuate for all coordinate components. The highest fluctuation is the $\mathrm{Y}$ coordinate difference, range from $-14 \mathrm{~cm}$ to $9 \mathrm{~cm}$, whereas $\mathrm{X}$-component is the most stable. However, the difference is up to $10 \mathrm{~cm}$ for $X$, but $7 \mathrm{~cm}$ for $Z$.

The station, 3D07, has the highest differences between the computed and existing VN-2000 coordinates. It may be caused by its lower quality of observation, in comparision with the others sites.

\subsection{Transformation from WGS84 to VN-2000}

The parameters for transformation from the VN-2000 to the WGS84 were determined based on the coordinate transformation parameters between $\mathrm{VN}-2000$ and ITRS as well as ITRF and WGS84 (shown in table 3).

Table 3. Coordinate transformation parameters between VN-2000 and WGS84

\begin{tabular}{|c|c|c|c|c|c|c|}
\hline $\begin{array}{c}\mathrm{T}_{1} \\
(\mathrm{~mm})\end{array}$ & $\begin{array}{c}\mathrm{T}_{2} \\
(\mathrm{~mm}) \\
\end{array}$ & $\begin{array}{c}\mathrm{T}_{3} \\
(\mathrm{~mm})\end{array}$ & $\begin{array}{c}\mathrm{D} \\
\left(10^{-9}\right) \\
\end{array}$ & $\begin{array}{c}\mathrm{R}_{1} \\
\text { (mas) }\end{array}$ & $\begin{array}{c}\mathrm{R}_{2} \\
\text { (mas) }\end{array}$ & $\begin{array}{c}\mathrm{R}_{3} \\
\text { (mas) }\end{array}$ \\
\hline \multicolumn{7}{|c|}{ WGS84 (G1762), epoch 2005.0} \\
\hline 194,7154 & 37,8661 & 110,4386 & $-2,2172$ & 1,0920 & 7,0395 & 72,1838 \\
\hline \multicolumn{7}{|c|}{ WGS84 (1674), epoch 2005.0} \\
\hline 194,7114 & 37,8691 & 110,4426 & $-9,1172$ & 1,3620 & 6,7695 & 72,5638 \\
\hline \multicolumn{7}{|c|}{ WGS84 (G1150), epoch 2001.0} \\
\hline 195,0293 & 38,01471 & 110,3792 & $-11,1682$ & 4,7517 & 1,4456 & 86,6502 \\
\hline \multicolumn{7}{|c|}{ WGS84 (G873), epoch 1997.0} \\
\hline 195,3391 & 38,15302 & 110,3148 & $-13,0293$ & 8,1414 & $-3,8783$ & 100,7366 \\
\hline \multicolumn{7}{|c|}{ WGS84 (G730), epoch 1994.0} \\
\hline 195,5565 & 38,24353 & 110,2624 & $-13,9225$ & 10,6837 & $-7,8713$ & 111,3614 \\
\hline \multicolumn{7}{|c|}{ WGS84 (origin), epoch 1984} \\
\hline 196,2898 & 39,11956 & 110,2802 & $-96,1000$ & 0,8579 & $-20,8810$ & 139,7773 \\
\hline
\end{tabular}

To evaluate the performance of these set of transformation parameters, station positions with respect to threre WGS84 reference frames, namely WGS84 (G1150), WGS84 (G1674) and WGS84 (G1762), were generated from two different ways. The first ways utilized the existing VN-2000 coorditantes and transformation parameters between VN-2000 and WGS84 that reprenseted in table 3 . The last one employed the known ITRF coorditantes and transformation parameters between ITRF and WGS84 that indicated in [14,28]. The results showed that WGS84 based coordinates derived from VN2000 coordinates is incoincide with corresponding quantities computed from the ITRF based coordinates. Statistical results shown in the table 4 reveals that the standard deviation of the coordinate difference between the two WGS84 derived coordinate values is the same level for all WGS84 reliazations in general. However, the standard deviation for WGS84 (G1674) and WGS84 (G1762) is small by comparision with WGS84 (G1150). The difference the standard deviation is at $1.5 \mathrm{~cm}$ and $2 \mathrm{~cm}$ level for GPS marine and Asia Pacific network, respectively. That could be explained by the fact that WGS84 (G1150) was introduced in 2001 [14], which is quite far from the measuring epochs (2015 and 2016) of the two GPS networks by comparision with the two new successive products, WGS84 (G1674) and WGS84 (G1762) published in 2012 and 2013, respectively.

In addition, the data in table 4 also indicated that the difference between the two WGS84 coordinate values for the Asia Pacific network is large by comparision with GPS marine network. The difference were at \pm 1.5 to $\pm 2 \mathrm{~cm}$ level in general. The standard deviation values were approximate $\pm 0.5 \mathrm{dm}, \pm 1 \mathrm{dm}$ and $\pm 0.4 \mathrm{dm}$ for $\mathrm{Z}, \mathrm{Y}$ and $\mathrm{Z}$ coodinates, respectively.

Table 4. The standard deviation between the two WGS84 coordinate values, one derived from ITRF coordinates and the other derived from VN-2000 coordinates

\begin{tabular}{|c|c|c|c|c|c|}
\hline \multirow{2}{*}{ Networks } & \multirow{2}{*}{$\begin{array}{l}\text { WGS84 } \\
\text { products }\end{array}$} & \multicolumn{4}{|c|}{ Standard deviation } \\
\hline & & $\sigma_{\mathrm{X}}(\mathrm{m})$ & $\sigma_{\mathrm{Y}}(\mathrm{m})$ & $\sigma_{\mathrm{Z}}(\mathrm{m})$ & $\sigma_{\mathrm{xyz}}(\mathrm{m})$ \\
\hline \multirow{3}{*}{$\begin{array}{c}\text { GPS } \\
\text { marine } \\
\text { network }\end{array}$} & $\begin{array}{c}\text { WGS84 } \\
\text { (G1150) }\end{array}$ & $\pm 0,0465$ & $\pm 0,1178$ & $\pm 0,0368$ & $\pm 0,0761$ \\
\hline & $\begin{array}{l}\text { WGS84 } \\
\text { (G1674) }\end{array}$ & $\pm 0,0463$ & $\pm 0,0913$ & $\pm 0,0330$ & $\pm 0,0621$ \\
\hline & $\begin{array}{c}\text { WGS84 } \\
\text { (G1762) }\end{array}$ & $\pm 0,0463$ & $\pm 0,0913$ & $\pm 0,0330$ & $\pm 0,0621$ \\
\hline \multirow{3}{*}{$\begin{array}{c}\text { Asia } \\
\text { Pacific } \\
\text { Network }\end{array}$} & $\begin{array}{c}\text { WGS84 } \\
\text { (G1150) }\end{array}$ & $\pm 0,0550$ & $\pm 0,1629$ & $\pm 0,0468$ & $\pm 0,1029$ \\
\hline & $\begin{array}{c}\text { WGS84 } \\
\text { (G1674) }\end{array}$ & $\pm 0,0503$ & $\pm 0,1206$ & $\pm 0,0362$ & $\pm 0,0783$ \\
\hline & $\begin{array}{l}\text { WGS84 } \\
\text { (G1762) }\end{array}$ & $\pm 0,0503$ & $\pm 0,1206$ & $\pm 0,0362$ & $\pm 0,0783$ \\
\hline
\end{tabular}

This paper also investigated the difference between WGS84 coordinates determined from VN-2000 coordinates based on existing transformation parameters published in [2] and ITRF2008 coordintes that considered as WGS84 coordinates because ITRF 2008 is agreement with WGS84 (G1674) at epoch 2005.0 [28]. The results in table 5 showed that the standard deviation between the computed and the known WGS84 coordinates values is significant. The standard deviation values were approximate $\pm 4 \mathrm{dm}, \pm 7 \mathrm{dm}$ and $\pm 3 \mathrm{dm}$ for $\mathrm{Z}, \mathrm{Y}$ and $\mathrm{Z}$ - axes, respectively. The standard deviation for position were at $\pm 5 \mathrm{dm}$ level that is quite large compared with the value of $\pm 0.07 \mathrm{dm}$ (shown in table 4). The huge difference reveals the new parameters outweight the existing ones published in [2] for transformation from VN-2000 to WGS84.

Table 5. The standard deviation between the WGS84 coordinates computed from VN-2000 coordinates based on existing transformation parameters and the known WGS84 coordinates

\begin{tabular}{|c|c|c|c|c|}
\hline \multirow{2}{*}{$\begin{array}{c}\text { GPS marine } \\
\text { Network }\end{array}$} & \multicolumn{4}{|c|}{ Standard deviation } \\
\cline { 2 - 5 } & $\sigma_{\mathrm{X}}(\mathrm{m})$ & $\sigma_{\mathrm{Y}}(\mathrm{m})$ & $\sigma_{\mathrm{Z}}(\mathrm{m})$ & $\sigma_{\mathrm{xyz}}(\mathrm{m})$ \\
\hline $\begin{array}{c}\text { GPS marine } \\
\text { Network }\end{array}$ & $\pm 0,3960$ & $\pm 0,7158$ & $\pm 0,3167$ & $\pm 0,5065$ \\
\hline $\begin{array}{c}\text { Asia Pacific } \\
\text { Network }\end{array}$ & $\pm 0,4483$ & $\pm 0,7057$ & $\pm 0,3510$ & $\pm 0,5235$ \\
\hline
\end{tabular}

\subsection{Transformation from PZ-90 to VN-2000}

The parameters for transformation from the VN-2000 to the WGS84 were determined based on the coordinate transformation parameters between VN-2000 and ITRS as well as ITRF and WGS84 (Table 6).

Similar to VN-2000 to WGS84 transformation, to evaluate the performance of these set of parameters, station positions with respect to PZ-90 reference frames, were yielded from two distinct solutions. The first 
deployed the available VN-2000 coorditantes and transformation parameters between VN-2000 andPZ-90 that shown in table 6 . The other one used existing ITRF coorditantes and transformation parameters between ITRF and PZ-90 that indicated in [16]. The results indicated that PZ-90 based coordinates derived from VN-2000 coordinates is incoincide with corresponding ones computed from the ITRF based coordinates. Statistical results shown in the table 7 reveals that the standard deviation of the coordinate differentiation between the two PZ-90 derived coordinate values is not the same level for all PZ- 90 versions. Th standard deviation for PZ-90.02 and PZ-90.11 is small by comparision with PZ -90.00. The difference the standard deviation is at $1.3 \mathrm{~cm}$ and $4.5 \mathrm{~cm}$ level for GPS marine and Asia Pacific network, respectively.

Table 6. Coordinate transformation parameters between VN-2000 and PZ-90

\begin{tabular}{|l|c|c|c|c|c|c|}
\hline $\begin{array}{c}\mathrm{T}_{1} \\
(\mathrm{~mm})\end{array}$ & $\begin{array}{c}\mathrm{T}_{2} \\
(\mathrm{~mm})\end{array}$ & $\begin{array}{c}\mathrm{T}_{3} \\
(\mathrm{~mm})\end{array}$ & $\begin{array}{c}\mathrm{D} \\
\left(10^{-9}\right)\end{array}$ & $\begin{array}{c}\mathrm{R}_{1} \\
(\mathrm{mas})\end{array}$ & $\begin{array}{c}\mathrm{R}_{2} \\
(\mathrm{mas})\end{array}$ & $\begin{array}{c}\mathrm{R}_{3} \\
(\mathrm{mas})\end{array}$ \\
\hline \\
\hline PZ 90.11, epoch 2010.0 \\
\hline 194,3132 37,6881 & 110,5373 & $-8,3285$ & $-2,8942$ & 13,4664 & 54,9538 \\
\hline PZ 90.02, epoch 2010.0 \\
\hline 193,9402 37,8741 & 110,7393 & $-16,3285$ & $-0,5942$ & 9,9264 & 59,1638 \\
\hline PZ 90.00, epoch 2002.0 \\
\hline 193,5202 & 38,0286 & 110,6000 & $-231,0905$ & 3,9042 & 2,7766 & 213,1286 \\
\hline
\end{tabular}

The data in table 7 also indicated that the discrepancy between the two PZ-90.00 coordinate values for the Asia Pacific network is large by comparision with GPS marine network. The standard deviation were at $\pm 1 \mathrm{dm}$ level for Asia Pacific network, but only were at $\pm 0.7 \mathrm{dm}$ level for GPS marine network . Conversely, the standard deviation for PZ-90.02 and PZ-90.11 coordinates for Asia Pacific network were slight smaller than GPS marine network. The standard deviation values $\pm 0.5 \mathrm{dm}, \pm 1 \mathrm{dm}$ and \pm $0.4 \mathrm{dm}$ for $\mathrm{Z}, \mathrm{Y}$ and $\mathrm{Z}$ coodinates, respectively.

Table 7. The standard deviation between the two PZ -90 coordinate values, one derived from ITRF coordinates and the other derived from $\mathrm{VN}-2000$ coordinates

\begin{tabular}{|c|c|c|c|c|c|}
\hline \multirow{2}{*}{ Networks } & PZ-90 & \multicolumn{5}{|c|}{ Standard deviation } \\
\cline { 3 - 6 } & products & $\sigma_{\mathrm{X}}(\mathrm{m})$ & $\sigma_{\mathrm{Y}}(\mathrm{m})$ & $\sigma_{\mathrm{Z}}(\mathrm{m})$ & $\sigma_{\mathrm{xyz}}(\mathrm{m})$ \\
\hline GPS & PZ-90.00 & $\pm 0,0445$ & $\pm 0,1049$ & $\pm 0,0342$ & $\pm 0,0687$ \\
\cline { 2 - 6 } marine & PZ-90.02 & $\pm 0,0530$ & $\pm 0,0738$ & $\pm 0,0340$ & $\pm 0,0560$ \\
Network & PZ-90.11 & & & & \\
\hline Asia & PZ-90.00 & $\pm 0,0536$ & $\pm 0,1523$ & $\pm 0,0440$ & $\pm 0,0966$ \\
\cline { 2 - 6 } Pacific & PZ-90.02 & $\pm 0,0488$ & $\pm 0,0707$ & $\pm 0,0271$ & $\pm 0,0520$ \\
Network & PZ-90.11 & & & \\
\hline
\end{tabular}

\section{Conclusion}

A vital segment of a nation's positioning infrastructure is the determination and maintenance of transformations from widely used global reference frames to the national geodetic datum. In this study, 14 transformation parameters between ITRF products and VN-2000 have been estimated using 10 stations located in Vietnam of network in the (APRGP) GPS campaign. The outcome of this study indicate that these parameters can be used to transform ITRF to VN-2000 with accuracy of $\pm 0.06 \mathrm{~m}$ or better, they therefore meet most the demands for average level of data accuracy. However, it is not the final answer to the problem, but rather an interim solution. For higher- precision applications, a generation of better transformation parameters between these systems is required.

Because it is difficult for determining WGS84 and PZ-90 coordinates with a high level of accuracy so the parameters for transformation from the VN-2000 to the WGS84 and PZ-90 were determined based on the coordinate transformation parameters between VN-2000 and ITRS. The test results showed that transformation parameters, on the basis of the results at the checkpoints, could generated station positions with the accuracy at several $\mathrm{cm}$ level for transformation from VN-2000 to the new realizations of WGS84 and PZ90 and reversely.

The results of the link between VN-2000 and ITRS, WGS84 and PZ-90 meeted the demand of practical tasks with medium accuracy requirements. However, in the future, the accuracy of these parameters will be improved with the addition of more accurate GPS coordinates in both the ITRS and VN-2000.

\section{Acknowledgments:}

The work presented in this paper was supported by a research grant from the Natural Resources and Environment of the Socialist Republic of Vietnam.

\section{REFERENCES}

1. Decision on the use of Vietnam's national references and coordinate datumm, No: 83/2000/QD-TTg of Prime Minister of Vietnam. Vietnam Government. No: 83/2000/QD-TTg. (2000)

2. Decision on the use of coordinate transformation parameters between international coordinate system 1984 (WGS-84) and Vietnamese coordinate datum (VN-2000), No. 05/2007/ QD-BTNMT of Minister of Vietnamese Natuaral resources and environmental Ministry Vietnam, Minister of Vietnam Natuaral resources and environmental Ministry No. 05/2007/QD-BTNMT. (2007)

3. C. Boucher and Z. Antamimi (2001). ITRS, PZ-90 and WGS 84: current realizations and the related transformation parameters. Journal of Geodesy, 75: 913-619.

4. G. Trần Bạch (2003). Introduction of Vietnam national reference and coordinate system (VN-2000). Department of Surveying, Mapping and Geoinformation of Vietnam, Vietnam

5. Z. Altamimi (2012), Role and importance of the International Terrestrial Reference Frame for sustainable development. Available on https://unstats.un.org/unsd/geoinfo/RCC/docs/rccap19 /Side\%20events/IAG-GGOS-ITRF-report2UNRRC10Oct2012.pdf

6. Z. Altamimi, X. Collilieux, J. Legrand, B. Garayt and C. Boucher (2007). ITRF2005: A new release of the International Terrestrial Reference Frame based on time series of station positions and Earth Orientation Parameters. J. Geophys. Res. Solid Earth, 112, B09401, doi:10.1029/2007JB00494

7. C. Boucher, Z. Altamimi and L. Duhem (1993). ITRF 92 and its associated velocity field, Technical Note 
15. Paris, France,Central Bureau of the IERS, Observatoire de Paris.

8. C. Boucher, Z. Altamimi and P. Sillard (1998). Results and analysis of the ITRF96, Technical Note 24. Paris, France, Central Bureau of the IERS, Observatoire de Paris.

9. C. Boucher, Z. Altamimi and P. Sillard (1999). The 1997 International Terrestrial Reference Frame (ITRF97), Technical Note 27. Paris, France, Central Bureau of the IERS, Observatoire de Paris

10. Z. Altamimi, P. Sillard and C. Boucher (2002). ITRF2000: A new release of the International Terrestrial Reference Frame for Earth science application. J. Geophys. Res. 107(B10), 2214, doi:10.1029/2001JB000561.

11. Z. Altamimi, X. Collilieux, J. Legrand, L. Laurent (2011) ITRF2008: an improved solution of the international terrestrial reference frame. Journal of Geodesy, Vol. 85(8), pp 457-473, doi:10.1007/s00190-011-0444-4

12. Z. Altamimi, R. Rebischung, L. Métivier and X. Collilieux (2016). ITRF2014: A new release of the International Terrestrial Reference Frame modeling nonlinear station motions, Journal of Geophysical Research. Journal of Geophysical Research, 121 (8): pp. 61096131, doi:10.1002/2016JB013098

13. http://lareg. ensg.ign.fr

14. Nga(2014). World geodetic System 1984. Its Definition and Relationships with Local Geodetic Systems.

15. S. Malys and J.A. Slater. Maintenance and Enhancement of the World Geodetic System 1984; Proceedings of ION GPS-94; Salt Lake City, Utah; September 1994.

16. http://eng.mil.ru/files/PZ-90.11_final-v8.pdf

17. Z. Altamimi, D. Angermann, D. Argus et al. (2001). The terrestrial reference frame and the dynamic Earth. Eos, Transactions, Am. Geophys. U., Vol. 82(25): p. 273-279.

18. J. Dawson and A. Woods. ITRF to GDA94 coordinate transformations. Journal of Applied Geodesy, 4:189-199, 2010. doi:10.1515/JAG.2010.019.

19. C. Boucher, Z. Altamimi (1993). Specifications for reference frame fixing in the analysis of a EUREF GPS campaign. Available on http://etrs89.ensg.ign.fr/memoV8.pdf.

20. S. Tomás (2014). Transformations between NAD83 (2011) and WGS84 (G1674). Available on https://www.ngs.noaa.gov/CORS/Articles/SolerWGS84 (G1674)toNAD83(2011).pdf

21. B. Graeme et al (2014). Reference Frames in Practice Manual, Commission 5 Working Group 5.2 Reference Frames,FIG Guide 2014.

22. C. Jekeli (2016). Geometric Reference Systems in Geodesy. Division of Geodesy and Geospatial Science, School of Earth Sciences, Ohio State University

23. Project (APRGP) GPS Campaign 2013. Record 2014/34. Geoscience Australia: Canberra.

24. G. Hu (2012). Report on the Analysis of the Asia Pacifc Regional Geodetic Project (APRGP) GPS
Campaign 2011. Record 2012/61. Geoscience Australia: Canberra.

25. G. Hu (2013). Report on the Analysis of the Asia Pacifc Regional Geodetic Project (APRGP) GPS Campaign 2012, Geoscience Australia.

26. G. Hu (2014). Report on the Analysis of the Asia Pacific Regional Geodetic

27. G. Hu (2016). Report on the Analysis of the Asia Pacific Regional Geodetic Project (APRGP) GPS Campaign 2015. Record 2016/20. Geoscience Australia, Canberra.

28. http://earth-info.nga.mil/GandG/sathtml 